\title{
EXAMINATION OF TEMPOROMANDIBULAR DISORDERS IN THE ORTHODONTIC PATIENT: A CLINICAL GUIDE
}

\author{
Ana Claúdia de Castro Ferreira CONTI ${ }^{1}$, Paula Vanessa Pedron OLTRAMARI ${ }^{1}$, \\ Ricardo de Lima NAVARRO ${ }^{1,2}$, Márcio Rodrigues de ALMEIDA ${ }^{1}$
}

1- DDS, MSc, PhD, Assistant Professor of Orthodontics, UNINGÁ Dental School, Maringá, PR, Brazil.
2- DDs, MSc, PhD, Assistant Professor of Oral and Maxillofacial Surgery, UNINGÁ Dental School, Maringá, PR, Brazil.

Corresponding address: Ana Cláudia de Castro Ferreira Conti - Rua Renato Tâmbara, 2-147 - Res. Samambaia, Cep.: 17018-100, Bauru-SP -phone/fax: +55 14 3223-5217 - e-mail: accfconti@uol.com.br

Received: November 17, 2006 - Accepted: February 12, 2007

\begin{abstract}
$T_{1}$

he possible association between orthodontic treatment and temporomandibular disorders (TMD) is a topic of great interest in the current literature. The true role of orthodontic therapy on the etiology of TMD, however, is still uncertain. From the clinical prospective, a thorough examination of the stomatognathic system is always necessary in order to detect possible TMD signs and symptoms prior to the beginning of the orthodontic therapy. Caution should be exercised when planning, performing and finalizing orthodontics, especially in patients who with history of signs and symptoms of TMD. The clinician must always eliminate patient's pain and dysfunction before initiating any type of orthodontic mechanics. Muscle incoordination, unstable disc-condyle relationship and bone alterations are usual TMD conditions that can interfere with the presenting occlusal relationship. This article reviews these aspects and presents a detailed clinical guide for the examination of the orthodontic patient, considering aspects related to facial pain and dysfunction.
\end{abstract}

Uniterms: Temporomandibular joint disorders; Orthodontics; Orthopedics; Dental occlusion.

\section{INTRODUCTION}

The possible relationship between orthodontic treatment and temporomandibular disorders (TMD) is frequently subject of discussion between clinicians and issue of different studies in the last decades $\mathrm{s}^{1,3,5,10,14,16,20-23}$. Despite these studies, many doubts concerning the real participation of orthodontic treatment in the etiology of TMD still remain unsolved. A thorough clinical interview and physical examination to detect TMD signs and symptoms prior to the establishment of the orthodontic therapy is mandatory. Even considering that orthodontic treatment does not cause TMD signs and symptoms ${ }^{27}$, caution should be exercised when planning, performing and finalizing orthodontics, especially in patients with a past history of signs and symptoms of TMD.

\section{ORTHODONTIC TREATMENT AND TMD}

Orthodontic therapy as a possible TMD etiologic factor has been a subject of discussion, especially after a lawsuit, in which orthodontic treatment was considered the main cause of pain $^{35}$. Thereafter, many studies in this field have been developed based on scientific data ${ }^{1,14,18}$.

Some authors have speculated that the deleterious effects of orthodontic mechanics in the stomatognathic system would be due to occlusal interferences or even to a new occlusal design, achieved after orthodontic therapy. Premolar extractions and incisor retraction, causing posterior displacement of the condyle and consequent overload to pain-sensitive areas used to be considered TMDcontributing factors as well $1^{9,25,28,42}$. This alteration in the condyle position would cause intra-capsular problems and joint pain. These statements, however, have been based merely on clinical experience and reports of personal points of view. Most scientific, evidence-based studies do not confirm these assumptions ${ }^{5,13,19}$.

\section{Orthodontics and TMD}

The role of functional and morphological malocclusion as a TMD-contributing factor has been widely discussed. The first report correlating occlusal factors and TMD symptoms is attributed to Costen ${ }^{7}$ in the 1930's. Since that time, different types of therapies involving orthodontic/ orthopedic treatment as well as occlusal adjustment have been proposed to correct malocclusion and improve TMD signs and symptoms ${ }^{8}$.

According to these theories, functional and morphological malocclusions cause TMD, and the achievement of an ideal occlusion through orthodontics or occlusal adjustment must eliminate pain and dysfunction. 
However, available longitudinal studies and well-designed statistical tests have shown that patients submitted to irreversible treatment frequently present relapse of TMD problems. Based on that, investigations concerning the role of occlusal and skeletal factors as contributors to TMD onset have been carried out.

Sadowsky and Begole ${ }^{38}$ (1980) reported that no relationship should be expected from orthodontic treatment and risks to develop TMD signs and symptoms. In a similar study, Conti, et al. ${ }^{5}$ (2003), evaluated the influence of orthodontic treatment on TMD etiology, comparing treated and untreated patients with malocclusion. Severe TMD was not found in the surveyed population, and no association between TMD severity and the type of orthodontic therapy was detected. The authors concluded that occlusion is considered a secondary factor in TMD etiology, which has a multifactorial aspect. Yet, TMD incidence was very similar in treated and untreated patients. It was also reported that orthodontic treatment has no relationship with TMD signs and symptoms when considering a successful orthodontic treatment. As orthodontics cannot cause TMD it also cannot be indicated to treat TMD.

According to McNamara ${ }^{26}$ (1997), the relationship between orthodontic treatment and TMD can be summarized in few topics:

1) TMD signs and symptoms may exist in healthy individuals;

2) TMD may develop during orthodontic treatment, but it does not cause TMD;

3) Orthodontic treatment performed during adolescence does not alter TMD risks;

4) There is no evidence that orthodontic mechanics can predispose the subject to a higher risk for TMD;

5) Even though the accomplishment of a stable occlusion is one of the orthodontic goals, TMD cannot be attributed to the failure in achieving this aim;

6) There is little evidence that orthodontic treatment can prevent TMD.

\section{Orthopedics and TMD}

Orthopedic treatment was first considered an etiologic factor of TMD because condyle position can be affected when mandibular protrusion is assumed with the use of orthopedic appliances. This type of therapy is worldwide used for correction of Class II in patients with mandibular deficiency.

Several studies ${ }^{30,31,41}$ have been conducted to evaluate TMD risks caused by the alterations in condyle position. Pancherz $^{32}$ (1985) reported an increase in muscular sensitivity in patients treated with mandibular repositioning appliances in the first 3 months. After 12 months these symptoms disappeared, which was explained based on the great level of TMJ adaptation. This finding is corroborated by Sfondrini, et al. ${ }^{39}$ (1996), who found an increase in muscle fibers resistant to fatigue and a decrease in muscle fibers sensitive to fatigue.

When considering condyle position, studies based on MRI findings before and after orthopedic treatment ${ }^{33,34,36,37}$ have demonstrated a tendency of condyle to return to its original position after the treatment is completed. It is worth mentioning that those reports do not consider the absence of condyle concentricity as a condition for joint health.

Even though anterior condyle position was partially maintained after orthopedic treatment with Herbst or Bionator appliances, this advanced mandibular position could improve joint pain in symptomatic subjects. This fact is due to the partial time repositioning appliances for these patients, which induce a retrodiscal adaptation, and an improvement of TMJ pain ${ }^{6}$.

To effectively deal with orthodontic patients, the professional should have a comprehensive knowledge of TMD, which would improve the quality of the treatment. Even considering that orthodontic treatment does not represent a great risk to develop TMD signs and symptoms, there is also no evidence that orthodontic treatment prevents TMD. Based on this, it is mandatory that the clinician performs a thorough examination before initiating any sort of rehabilitation treatment, such as orthodontic therapy.

\section{Patient examination}

For most patients, the examination process includes a detailed clinical interview and a comprehensive physical inspection. Temporomandibular joint (TMJ) imaging and additional tests (as serology and electromyography) are necessary only for very few specific cases. It has been stated that approximately $70 \%$ of the diagnostic process is based on the history review ${ }^{3}$. Physical examination must include investigation of the mandibular active range of motion (AROM), standardized TMJ and masticatory and cervical muscle palpation, as well as inspection of articular joint sounds. In case of any abnormality, the orthodontist should refer the patient to a TMD specialist to perform TMD management prior to the starting the orthodontic therapy.

The clinical interview of the TMD patient should be well documented and must contain questions regarding the onset of the problem, previous diagnosis and performed treatment $t^{2,24,29}$.

\section{Anamnesis}

The following information should be part of a comprehensive history: chief complaints, history of present illness, past medical and dental history, review of the systems (systemic conditions that can enhance or cause the pain sensation) and psychosocial history.

History review is the most important part of the examination process. The first question to be done is about the chief complaint, which is the main reason that made the patient seek help. This information is of great importance because even if the patient has many complaints, the attenuation or resolution of the main problem may improve the general status and quality of life ${ }^{2,24,29}$.

Each complaint should be listed separately in order of importance to the patient, and shall contain information about:

- Onset: it relates to when the patient first noticed the symptoms and is important in order to define for how long 
the patient has been sick. This information is useful to determine whether the patient has an acute or chronic condition, which is crucial for the establishment of a proper therapy.

- Location: the patient should be oriented to indicate with only one finger the exact site of his/her pain. The intracapsular pain is well pointed by the patient, but muscle pain is diffuse and difficult to be localized. The detection of the pain source is decisive for the success of the treatment. It is important to note that the site of pain can be different from the source of pain (ectopic pain), as in the myofascial pain syndromes.

- Intensity: Intensity of pain is a difficult parameter to quantify. The visual analogue scale (VAS) is a simple and reliable method that is extensively used in clinical practice and research to measure pain intensity. It is a visual representation of relative pain intensity consisting of a 10cm horizontal line with "no pain" at one end and "worst pain ever" at the opposite end. By simply placing a mark along this line, the patient is able to display his/her relative pain intensity.

- Frequency: it is known as its temporal behavior. The patient is asked whether the pain is constant or paroxysmal, which means that it comes in periods of attacks. Constant pain will obviously require an immediate care. When pain is of musculoskeletal origin and manifests only during activities such as chewing and speaking, the treatment normally assumes a non-invasive approach. Pain that comes in quick attacks and lasts for seconds is usually related to either trigeminal or glossopharyngeal neuralgia.

- Quality: Patients are often not able to determine exactly the quality of pain they are suffering. TMD pain is normally described as deep, dull and sometimes aching (throbbing), like in the inflammatory acute processes of the joints. Burning or shock-like pain is probably from neuropathic origin. Headache reports are associated with migraine or other primary headache disorders.

- History of the chief complaints: it is valuable to detect possible aggravating factors to the pain and to obtain more information about the patient's chief complaints. Musculoskeletal pain is aggravated when using masticatory system structures and also by emotional stress. Avoiding these activities or using antiinflammatory or analgesic medications may alleviate patient symptoms. Vascular or neurogenic pain is usually not affected by masticatory function. The orthodontist should also ask patients about previous treatment modalities, traumatic events and mode of pain onset.

- Current and past medications: If the patient is taking any medication, it must be reported because some conditions can be associated with drug side effects. Additionally, drugs that will be possibly prescribed can interact with those that the patient is already taking. Questions regarding allergies are also very important.

- Medical and surgical history: Questions related to general health conditions must be answered by the patient. Some systemic pathologies, such as fibromyalgia and osteoarthritis, among others, can cause generalized pain and dysfunction.

- Family history: The patient should report if some relative presents the same conditions because some disorders are genetically predisposed. Migraine, for instance, is a primary headache related to family inheritance.

- Dental history: many patients associate the onset of the painful sensation with a procedure performed by a dentist. Patients very often report the onset of pain after long dental treatment appointments, such as root canal therapy and third molar extractions.

- Presence of parafunctional habits: The patient should be asked about the presence of any parafunctional activity. The habits most frequently found in TMD patients are clenching and grinding. Nail biting and poor posture due to occupational activities should also be recorded.

\section{Physical examination}

At this point, the clinician should have a reasonable idea of the nature of patient's problem. A comprehensive physical examination will help to determine the source of pain as well as the severity of the dysfunction. This part of assessment includes TMJ evaluation (joint range of motion, inspection of joint sounds and pain on palpation), and muscle palpation. Additional diagnostic tests can be necessary for some patients. Dental and occlusal evaluations are also performed $^{2,4,29}$.

\section{I- TMJ evaluation}

TMJ clinical inspection is often based on joint range of motion, pain on palpation and presence of joint sounds during mandibular and opening movement.

TMJ range of motion: some chief complaints include limitation of opening and difficulties in mandibular movement. The patient is requested to fully open the mouth and the sum of interincisal distance and overbite, measured with a millimeter rule is documented (Figure 1). The normal values to maximum opening range from 45 to $55 \mathrm{~mm}^{11}$, although smaller figures are frequently found in asymptomatic individuals. The mandibular opening and closing movements may be accomplished in a straight line, to assess deviation or deflection. Measurements of protrusion, lateral right and left movements must also be performed. For these measurements it is recommended the demarcation of two reference points, on the maxilla and mandible, close to the midline. These reference points will assist the measurements of the range of motion during mandibular excursion (Figure 1).

- Detection of joint sounds: The presence of joint sounds during mouth opening and mandibular excursion can be useful in the diagnosis of disc-condyle incoordination. It is believed that the clinical registration by means of manual inspection or by using a stethoscope is very reliable in the detection of articular sounds ${ }^{11}$ (Figure 2). Clicking, crepitation and terminal thud (related to hypertranslation) are the most common sounds in TMD patients.

- TMJ palpation: Tenderness to palpation is considered one of the most important signs in the detection of intracapsular pathologies. During repeated opening and 
closing movements the clinician should locate the lateral polo of mandibular condyle. After that, with the patient maintaining the mouth in a relaxed position, TMJ bilateral and simultaneous palpation of the lateral aspect of the joint should be done. This palpation should be performed with pressure of $1 \mathrm{kgf}$ in the lateral and posterior aspects of the joints (Figure 3). Reports of pain can lead to diagnosis of capsulitis and/or sinovitis. In order to graduate the patient's response to palpation, score ranging from 0 to 3 can be used: 0 - absence of pain on palpation; 1 - mild pain; 2 moderate pain; 3 - severe pain, palpebral reflex or "jump sign" $", 12$.

\section{II- Muscle palpation}

Muscle palpation is a very important step in the diagnosis of TMD and myofascial pain syndromes. By means of mechanical stimuli caused by digital pressure, nociceptive neurons located in the muscular and myofascial structures are stimulated to detect and transmit pain messages to the central nerve system. The graduation of patient's response to palpation allows evaluating the severity of pain and is used to measure the efficacy of a given treatment modality in follow-up visits. Palpation should be performed with a pressure of $1.5 \mathrm{Kg}$, which is strong enough to elicit pain message in symptomatic patients, and mild enough to not cause pain in asymptomatic control subjects $^{2,15,40}$.

Palpation should be done bilaterally, in a relaxed position, with the tip of the finger or by pincer palpation, when no underline bone support is present. Yet, during the examination, the patient should be seated facing the orthodontist in such a way that the clinician can observe the patient's reactions.

The three portions of the temporalis (posterior, medial and anterior) (Figure 4), superficial and deep masseter (Figure 5 ), as well as the insertion of the medial pterygoid muscle should be examined. The sternocleidomastoid, supeior trapezius and subocciptal are important cervical muscles to be also considered in this evaluation.

Muscle palpation is also scored 0 to 3 , according to the patient's response ${ }^{34}$. The detection of trigger points in the myofascial structures is done during the examination. When the patient presents severe pain, this spot is continuously pressed from 8 to 10 seconds in order to stimulate referred pain. When referred pain zones are reproduced, a diagnosis of myofascial pain is done, which requires specific management modalities.

\section{III- Dental and occlusal evaluation Dental examination}

Dental and periodontal conditions, such as defective restorations, missing teeth or periodontal problems that could contribute to pain onset should be detected at this moment. Most orofacial pain conditions has a dental origin ${ }^{17}$. The presence of incisal or occlusal dental attrition is also an indicator of possible parafunctional habits.

\section{Occlusal examination}

The presence or absence of lateral and anterior guides (Figure 6) is recorded as the overbite and overjet. In this evaluation, the patient is asked to perform lateral mandibular movements in order to detected occlusal interferences in the non-working side, using a cellophane paper. The discrepancies between centric relation and intercuspal position are also registered by means of the mental pressure technique. When large discrepancies are detected or the results are uncertain, an articulator mounting can be indicated ${ }^{2}$.

\section{IV - Additional Diagnostic Tests ${ }^{2}$}

In case some doubt still persists, additional tests can help defining a diagnostic impression. Functional muscle manipulation, TMJ overloading, cryotherapy and diagnostic nerve blockage are useful for this purpose.

\section{V- TMJ imaging assessment}

The real need and validity of TMJ images in the diagnosis of TMD is controversial, despite all technological apparatus available in present days. Joint imaging should be indicated based on the dentist's good sense, but diagnosis and treatment techniques are still mainly elaborated based on clinical examination ${ }^{24}$.

The general rule is that imaging exams are necessary when they might, somehow, change an initially established management strategy. The overestimation of image findings, followed by unnecessary irreversible treatment is a potential problem, especially for non-experienced clinicians.

Panorex is helpful only to rule out dental and bone pathologies, with no validity on the diagnosis of TMJ position or anatomical form. Transcranial, lateral images and computed tomography can detect bone changes, condyle degeneration, mobility and fractures. Magnetic resonance image (MRI), on the other hand, is able to detect TMJ disc position and the presence of inflammatory processes.

Again, the detection of small abnormalities in TMJ images is highly prevalent in asymptomatic individuals and does not mean that a treatment is mandatory. Flattening of the condyle in older subjects is an example of this statement.

\section{CONCLUSION}

The available evidence-based data demonstrate that orthodontic treatment has little to do with TMD signs and symptoms. Some conditions, such as muscle incoordination, unstable disc-condyle relationship and bone alterations can interfere with the occlusal relationship and interfere with orthodontic analysis. A non-invasive approach and reversible treatment of the TMD conditions are mandatory for all patients before the orthodontic therapy starts. In case of relapse of symptoms during the course of orthodontics, the patient should be reexamined and, if necessary, mechanics should be discontinued until the improvement of TMD signs and symptoms. 


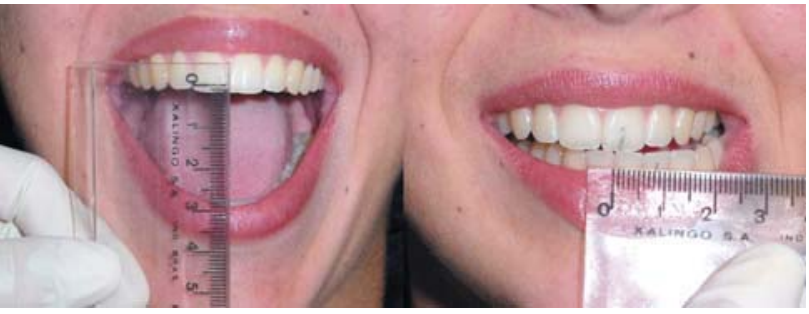

FIGURE 1- Measurement of maximum active opening and maximum lateral movement

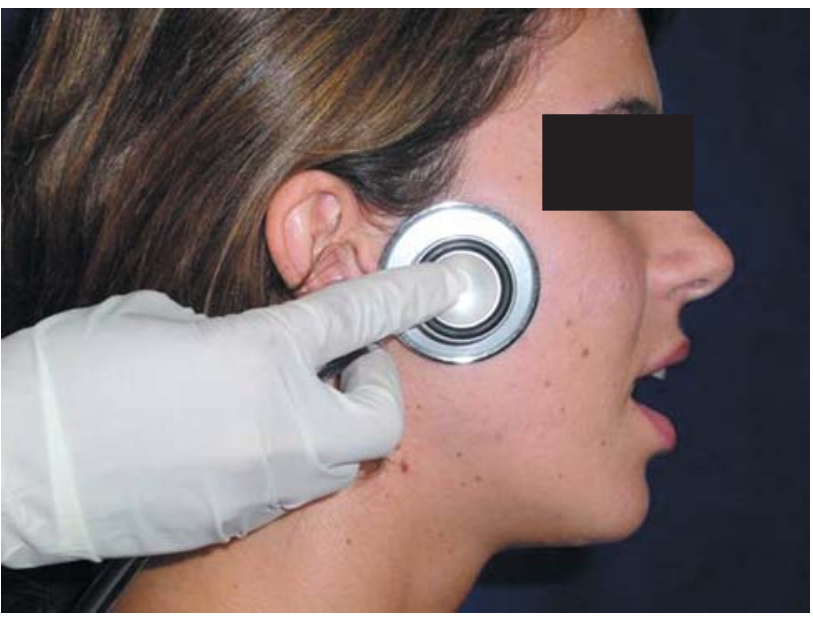

FIGURE 2- Joint sound inspection with a stethoscope

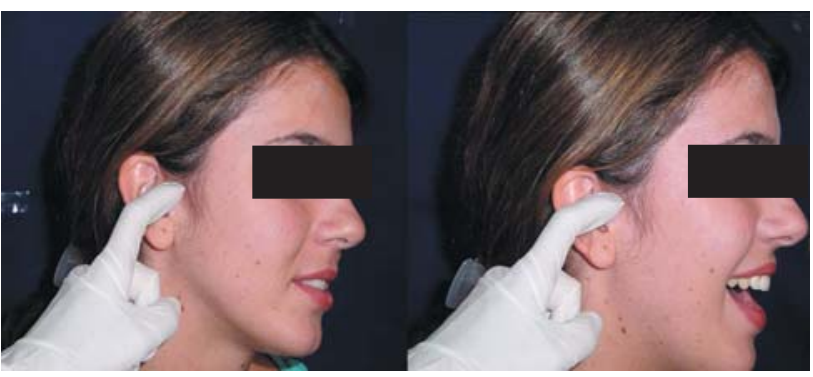

FIGURE 3- Palpation of TMJ's lateral and posterior aspects

\section{REFERENCES}

1- Artun J, Hollender LG, Truelove EL. Relationship between orthodontic treatment, condylar position, and internal dearangement in the temporomandibular joint. Am J Orthod Dentofacial Orthop. 1992;101(1):48-53.

2- Austin DG, Peters RA. Examination of the TMD patient. In: Peters RA, Gross SG. Clinical management of temporomandibular disorders and orofacial pain

Chicago: Quintessence; 1995. p. 123-60.

3- Beattie JR, Paquete DE, Johnston LE Jr. The functional impact of extraction and nonextraction treatments: a long-term comparison in patients with "borderline", equally susceptible Class II malocclusion. Am J Orthod Dentofacial Orthop. 1994;105(3):444-9.

4- Clark GT. Examining temporomandibular disorders patients for craniocervical dysfunction. J Craniomandibular Pract. 1984;2(1):5563.

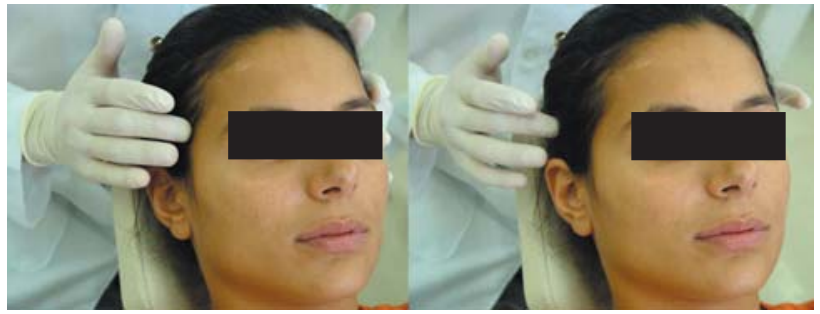

FIGURE 4- Palpation of anterior and posterior temporalis muscle

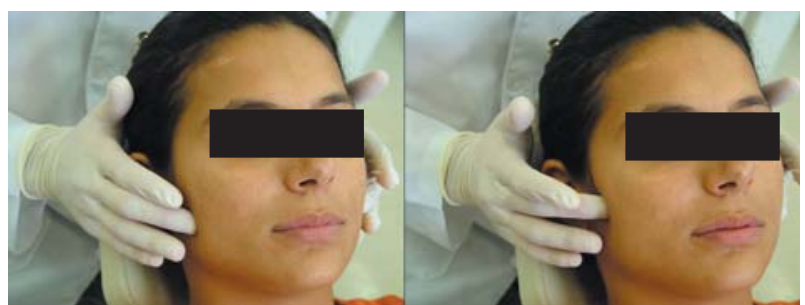

FIGURE 5- Palpation of the superficial and deep masseter muscle

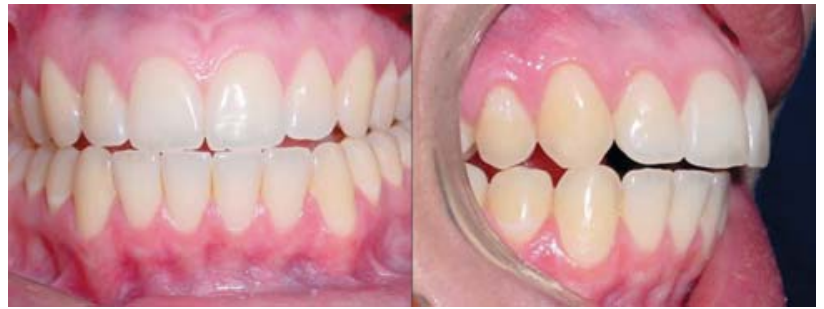

FIGURE 6- Anterior and lateral guide assessment

5- Conti ACCF, Freitas MR, Conti PCR, Henriques JFC, Janson GRP. Relationship between signs and symptoms of temporomandibular disorders and orthodontic treatment: a cross-sectional study. Angle Orthod. 2003;73(4):411-7.

6- Conti PCR, Santos CN, Kogawa EM, Conti ACCF, Araújo CRP. The treatment of painful temporomandibular joint clicking with oral splints: a randomized clinical trial. J Am Dent Assoc. 2006;137(8):1008-14

7- Costen JB. A syndrome of ear sinus symptoms dependent upon disturbed functions of TMJ. Ann Otol. 1934;43(1):1-15.

8- Costen JB. Neuralgias and ear symptoms associated with disturbed function of the TMJ. J Am Med Assoc. 1936;107:252-4.

9- Covey EJ. The effects of bicuspids extraction orthodontics on TMJ dysfunction. Funct Orthod. 1990;7(3):1-2.

10- Dibbets JMH, VanDerWeele LTH. Signs and symptoms of temporomandibular disorders (TMD) and craniofacial from. Am J Orthod Dentofacial Orthop. 1996;110(1):73-8.

11- Dworkin SF, Huggins KH, LeResche L, Von Korff M, Howard J, Truelove E, et al.. Epidemiology of signs and symptoms in temporomandibular disorders: clinical signs in cases and controls. J Am Dent Assoc. 1990;120(3):273-81. 
12- Dworkin SF, LeResche L. Research diagnostic criteria for temporomandibular disorders: review, criteria, examinations and specifications, critique. J Craniomandib Disord. 1992;6(4):301-55.

13- Egermark I, Magnusson T, Carlsson GE. A 20 year follow-up of signs and symptoms of temporomandibular disorders and malocclusion in subjects with or without orthodontic treatment in childhood. Angle Orthod. 2003;73(2):109-15.

14- Egermark I, Thilander B. Craniomandibular disorders with special reference to orthodontic treatment: an evaluation from childhood to adulthood. Am J Orthod Dentofacial Orthop. 1992;101(1):28-34.

15- Fischer AA. Pressure threshold measurement for diagnosis of myofascil pain and evaluation of treatment results. Clin J Pain. 1987:2:207-14

16- Gianelly AA, Anderson CK, Boffa J. Longitudinal evaluation of condylar position in extraction and nonextraction treatment. Am J Orthod Dentofacial Orthop. 1991;100(5):416-20

17- Graff-Radford SB, Solberg WK. Atypical odontalgia. J Craniomandib Disord. 1992;6(4):260-5.

18- Henrikson T, Nilner M, Kurol J. Symptoms and signs of temporomandibular disorders before, during and after orthodontic treatment. Swed Dent J. 1999;23(5-6):193-207.

19- How CK. Orthodontic treatment has little to do with temporomandibular disorders. Evid Based Dent. 2004; 5(3):75. 20- Katzberg RW, Westesson PL, Tallents RH, Drake CM. Orthodontics and temporomandibular joint internal dearangement. Am J Orthod Dentofacial Orthop. 1996;109(5):515-20.

21- Kundinger KK, Austin BP, Christensen LV, Donegan SJ, Ferguson DJ. An evaluation of temporomandibular joints and jaw muscles after orthodontic treatment involving premolar extractions. Am J Orthod Dentofacial Orthop. 1991;100(2):110-5.

22- Lagerström L, Egermark I, Carlsson GE. Signs and symptoms of temporomandibular disorders in 19-year-old individuals who have undergone orthodontic treatment. Swed Dent J. 1998; 22(5-6):17786

23- Larsson E, Ronnerman A. Mandibular dysfunction in orthodontically treated patients ten years after the completions of treatment. Eur J Orthod. 1981;3:89-94.

24- Laskin DM, Greene CS, Hylander WL. TMD's and evidencebased approach to diagnosis and treatment. Chicago: Quintessence; 2006.

25- Loft GH, Reynolds JM, Zwemer JD, Thompson WO, Dushku J. The occurence of craniomandibular symptoms in healthy young adults with or without prior orthodontic treatment. Am J Orthod Dentofacial Orthop. 1989;96(3):264-5.

26- McNamara JA Jr. Orthodontic treatment and temporomandibular disorders. Oral Surg Oral Med Oral Pathol. 1997;8(1):107-17.

27- Mcnamara JA Jr, Selligman DA, Okeson JP. Occlusion orthodontic treatment and temporomandibular disorders: a review. J Orofac Pain. 1995;9(1):73-90

28- Nielsen L, Melsen B, Terp S. TMJ function and the effects on the masticatory system on 14-16-year-old Danish children in relation to orthodontic treatment. Eur J Orthod. 1990;12(3):254-62.

29- Okeson JP. History and examination for temporomandibular disorders. In: Okeson JP. Management of temporomandibular disorders and occlusion. Saint Louis: Mosby-Year Book; 1993. p. 245-320.
30- Owen AH. Orthodontic/orthopedic therapy for craniomandibular pain dysfunction. Part B. Treatment flow, sheet, anterior disk displacement, and case histories. Cranio. 1988;6(1):48-63.

31- Owen AH. Unexpected TMJ responses to functional jaw orthopedic therapy. Am J Orthod Dentofacial Orthop. 1988;94(4):338-49.

32- Pancherz H. The Herbst appliance: its biologic effect and clinical use. Am J Orthod Dentofacial Orthop. 1985;87(1):1-20.

33- Pancherz H, Ruf S, Kohlhas P. Effective condylar growth and chin position changes in Herbst treatment: a cephalometric roentgenographic long term study. Am J Orthod Dentofacial Orthop. 1998;114(4):427-46.

34- Pancherz H, Ruf S, Thomalske-Faubert C. Mandibular articular disc position changes during Herbst treatment: a prospective longitudinal MRI study. Am J Orthod Dentofacial Orthop. 1999;116(2):207-14.

35- Pollack B. Cases of note. Michigan Jury Awards $\$ 850.000$ in ortho case: a tempest in teapot. Am J Orthod Dentofacial Orthop. 1998;94:358-9.

36- Ruf S, Pancherz H. Long-term TMJ effects of Herbst treatment: a clinical and MRI study. Am J Orthod Dentofacial Orthop. $1998 ; 114(5): 475-83$.

37- Ruf S, Pancherz H. Temporomandibular joint growth adaptation in herbst treatment: a prospective magnetic resonance imaging and cephalometric roentgenographic study. Eur J Orthod. 1998;20(4):37588

38- Sadowsky C, BeGole E. Long-term status of temporomandibular joint function and functional occlusion after orthodontic treatment. Am J Orthod. 1980;78(2):201-12.

39- Sfondrini G, Reggiani C, Gandini P, Bovenzi R, Pellegrino MA. Adaptations of masticatory muscles to a hyperpropulsive appliance in the rat. Am J Orthod Dentofacial Orthop. 1996;110(6):612-7.

40- Silva RS, Conti PCR, Lauris JRP, Silva ROF, Pegoraro LF. Pressure pain threshold in the detection of masticatory myofascial pain: an algometer-based study. J Orofac Pain. 2005;19(4):318-24.

41- Witzig J, Spahl TJ. The clinical managment of basic maxilloafacial orthopedic appliances. Hong Kong: Year Book Medical; 1987.

42- Wyatt W. Preventing adverse effects on the temporomandibular joint through orthodontic treatment. Am J Orthod Dentofacial Orthop. 1987;91(6):493-9. 